\title{
Low temperature micro-photoluminescence spectroscopy of microstructures with InAsP/InP strained quantum wells
}

\author{
J P Landesman ${ }^{1}$, N Isik-Goktas ${ }^{2}$, R R LaPierre ${ }^{2}$, C Levallois ${ }^{1}$, S \\ Ghanad-Tavakoli $^{2}$, E Pargon ${ }^{3}$, C Petit-Etienne ${ }^{3}$ and J Jiménez ${ }^{4}$ \\ ${ }^{1}$ Univ Rennes, INSA Rennes, CNRS, Institut FOTON-UMR 6082, F-35000 \\ Rennes, France \\ ${ }^{2}$ Department of Engineering Physics, McMaster University, Hamilton, ON L8S 4L7, \\ Canada \\ ${ }^{3}$ Univ. Grenoble Alpes, CNRS, CEA/LETI-Minatec, Grenoble INP, LTM, F-38054 \\ Grenoble-France \\ ${ }^{4}$ Dpto. Fisica de la Materia Condensada, Universidad de Valladolid, 47011 \\ Valladolid, Spain \\ E-mail: Jean-Pierre.Landesman@univ-rennes1.fr
}

April 2021

\begin{abstract}
Ridge microstructures were prepared by reactive ion etching (RIE) of a series of stacked $\operatorname{InAs}_{x} \mathrm{P}_{1-x}$ quantum wells (QWs) with step graded compositions grown on InP by molecular beam epitaxy. These microstructures were characterized by low temperature micro-photoluminescence. The photoluminescence (PL) emission associated with each of the QWs was clearly identified and a model for their line shape was implemented. PL line-scans were measured across etched ridge stripes of various widths in an optical cryostat, with a spatial resolution of $1 \mu \mathrm{m}$. The model for the PL spectra allowed accurate extraction of the local PL integrated intensities, spectral positions and line widths. Two different RIE processes, using $\mathrm{CH}_{4} / \mathrm{H}_{2}$ and $\mathrm{CH}_{4} / \mathrm{Cl}_{2}$, were investigated. The PL line-scans showed strong variations of the integrated PL intensities across the etched stripes. The PL intensities for all QWs increased gradually from the edge to the center of the ridge microstructures, over a length scale of 10 to $20 \mu \mathrm{m}$. On the other hand, the spectral peak position of the PL lines remained constant ( 0.2 to $0.4 \mathrm{meV}$, depending on which $\mathrm{QW}$ was considered) across the microstructures. These observations are discussed in terms of the mechanical stress induced by the RIE processes, the relaxation of the biaxial built-in compressive stress in the InAsP QWs (induced by the free surfaces at the vertical etched sidewalls), and also by the non-radiative recombination at these sidewalls. Altogether, this study illustrates the contribution that specially designed test structures, coupled with advanced spectroscopic characterization, can provide to the development of semiconductor photonic devices (e.g. lasers or waveguides) involving RIE processing.
\end{abstract}

Keywords: $\operatorname{InAs}_{x} \mathrm{P}_{1-x} / \mathrm{InP}$ quantum wells, micro-PL, reactive ion etching

Submitted to: J. Phys. D: Appl. Phys. 


\section{Introduction}

Given the amount of published work based on nanostructures, there is still a need for tools and methodologies to accurately determine material properties at local scales. Characterizing and understanding the optical properties on a local scale of two-dimensional, one-dimensional and zero-dimensional semiconductor heterostructures deserves specific attention. The confinement geometry by itself is a source of modification of the local optical properties [1], but the synthesis technique as well as the processing of the material to elaborate micro- and nanostructures may also have nonnegligible impact on the material properties [2]. For example, the photoluminescence (PL) signal measured from quantum well (QW) samples patterned into micro- or nanoobjects may differ from that of the as-grown QW material [3]. Processing of QW samples to form building blocks for photonic devices, such as ridge waveguides, may also affect the PL properties [4]. In this work, we investigate the changes in the PL emission from $\operatorname{InAs}_{x} \mathrm{P}_{1-x}$ QWs when ridge structures are fabricated by reactive ion etching (RIE). Low temperature micro-PL $(\mu \mathrm{PL})$ allows us to identify different trends, indicating that the luminescence emission of the QWs is sensitive to the etching process. In particular, the local PL intensity is affected by the mechanical stress within the stripes. First, as was demonstrated recently, the RIE process itself can introduce some mechanical stress, probably due to diffusion and implantation of ions and reactive radicals near the etched sidewalls [5]. Secondly, as the $\operatorname{InAs}_{x} \mathrm{P}_{1-x}$ QWs were grown with compressive built-in stress, stress relaxation occurs near the etched sidewalls which are free surfaces. Moreover, the local PL intensity may also be affected by non-radiative recombination due to creation of the etched sidewalls and the change in the surface recombination velocity (SRV) resulting from the etching process. The information produced by such $\mu \mathrm{PL}$ experiments can be useful from two points of view. First, it helps to understand the fundamentals of the interactions of etching plasmas with the semiconductor material. This remains still an active field of research, particularly because new plasma chemistries and etching processes are being used $[6,7,8,9]$. The specific design of our graded QW samples contributes to improving the knowledge gained on this kind of interaction. Secondly, it can provide a methodology to better monitor the perturbation undergone by the active material during processing of photonic structures such as waveguides,

especially when the critical dimension of these structures is reduced and the device performance can be affected by the material changes or degradation. Altogether, the evolution of the PL intensity can be an indicator of possible damage to the material during the processing of photonic devices which can limit the performance of components such as laser diodes or passive waveguides.

\section{Sample preparation}

The sample structure is illustrated in figure 1. The As composition in the QWs is step graded, with QW8 (closest to the substrate) having the highest As composition 


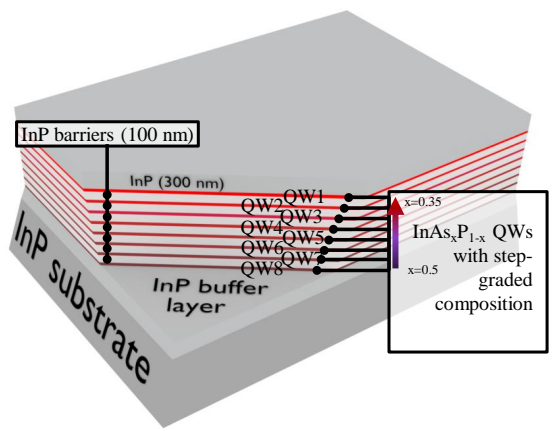

Figure 1. Epitaxial sample structure. Each $\operatorname{InAs}_{x} \mathrm{P}_{1-x}$ QW has the same thickness $(7.5 \mathrm{~nm})$. The $\mathrm{As} /(\mathrm{P}+\mathrm{As})$ composition is step graded, from 0.5 for QW8 to 0.35 for QW1.

and QW1 (closest to the surface) having the lowest As composition. This controls the energy gap of the different QWs such that the PL emission from the deeper QWs is not absorbed as it travels through the upper-lying QWs, which are transparent to this PL emission. The sample was grown by gas source molecular beam epitaxy (MBE) on an ntype InP (100) substrate using a solid source for In and a gas cracker for $\mathrm{PH}_{3} / \mathrm{AsH}_{3}$ gases (producing $\mathrm{P}_{2} / \mathrm{As}_{2}$ ). The growth temperature was $470{ }^{\circ} \mathrm{C}$. The $\mathrm{InP}$ buffer thickness was 300 to $400 \mathrm{~nm}$. No dopant was included during growth of the buffer and QW structure. The design included a constant thickness for the QWs, made possible by keeping the In flux and the duration of each QW growth sequence constant. The QW thickness was $7.5 \mathrm{~nm}$. Careful calibration of the As and $\mathrm{P}$ sources, prior to sample growth, yielded a linear variation of the As to $\mathrm{P}$ composition ratio along the $8 \mathrm{QWs}$. The As/(P+As) atomic composition was 0.5 for QW8 and 0.35 for QW1.

Microstructures made of ridges with a rectangular cross-section were then etched on these samples. A silicon nitride $\left(\mathrm{SiN}_{x}\right)$ mask thin film (500 nm thick) was first deposited by plasma-enhanced chemical vapor deposition at $250^{\circ} \mathrm{C}$. Stripes of various widths, from 1 to $50 \mu \mathrm{m}$, and a length of several $\mathrm{mm}$, were defined by optical lithography in this mask. Finally, the ridges were fabricated by RIE using two different processes. In process \# 1 , a capacitively coupled RIE reactor (Alcatel 300) with a chamber $300 \mathrm{~mm}$ in diameter was used. The gaseous species involved are $\mathrm{CH}_{4}, \mathrm{H}_{2}$, and Ar with the following respective flow rates: $6.4 \mathrm{sccm} / 50 \mathrm{sccm} / 8.4 \mathrm{sccm}$. The total pressure was maintained at 30 mTorr during the process and the applied RF power was $40 \mathrm{~W}$ leading to a DC self-bias of $360 \mathrm{~V}$. Under these conditions a moderate etch rate of $20 \mathrm{~nm} / \mathrm{min}$ is reached but smooth sidewalls are obtained for the patterns formed. As this etching rate is low, to avoid heating during long etching steps, samples were attached to a water-cooled holder (water temperature between 10 and $15{ }^{\circ} \mathrm{C}$ ). In process \# 2, an industrial inductively coupled plasma reactor accepting $200 \mathrm{~mm}$ wafers (DPS+ from Applied Materials) with $\mathrm{Cl}_{2} / \mathrm{CH}_{4} / \mathrm{Ar}$ gas mixture was used [10]. This reactor is equipped with a hot cathode whose temperature can be tuned from $150{ }^{\circ} \mathrm{C}$ to $250{ }^{\circ} \mathrm{C}$. The reactor chamber walls are coated with $\mathrm{Al}_{2} \mathrm{O}_{3}$. The chamber walls are kept at $80^{\circ} \mathrm{C}$ and the ceramic dome is 
maintained at $60{ }^{\circ} \mathrm{C}$. The III-V samples are thermally glued with a thermal paste on a $200 \mathrm{~mm} \mathrm{SiO}_{2}$ carrier wafer. The plasma conditions used in this study were : $20 \mathrm{sccm}$ $\mathrm{Cl}_{2} / 100 \mathrm{sccm} \mathrm{Ar} / 32 \mathrm{sccm} \mathrm{CH}_{4}$, pressure chamber 20 mTorr, source power $800 \mathrm{~W}$, bias power $200 \mathrm{~W}$, chuck temperature $200{ }^{\circ} \mathrm{C}$. The resulting etch rate was $900 \mathrm{~nm} / \mathrm{min}$. The samples were etched to a depth of $2 \mu \mathrm{m}$ to ensure the etching of all the QWs. After the semiconductor etching process, the remaining $\mathrm{SiN}_{x}$ mask was etched away using a solution of hydrofluoric (HF) acid.

\section{Low temperature micro-photoluminescence measurements}

The $\mu \mathrm{PL}$ experiments were performed at $10 \mathrm{~K}$ in an optical cryostat. Details of the set-up have been previously reported [11]. The excitation wavelength was $1064 \mathrm{~nm}$, with a spot size of approximately $1 \mu \mathrm{m}$. This wavelength generates electron-hole pairs in the $\operatorname{InAs}_{x} \mathrm{P}_{1-x}$ QWs but not in the InP buffer, substrate and barrier layers. The excitation power density was approximately $10^{6} \mathrm{~W} / \mathrm{cm}^{2}$ but a small part of this pump power is used for the QWs excitation since absorption occurs only in the QWs. $\mu \mathrm{PL}$ line-scans across the etched ridges could be performed with a step size of $5 \mu \mathrm{m}$, using high accuracy piezo-electric nano-positioners on the sample holder inside the cryostat. The spectrometer resolution was $0.025 \mathrm{~nm}$. Figure 2 illustrates the PL spectrum from the as-grown sample. Eight emission lines can be clearly identified. A simple best-fit
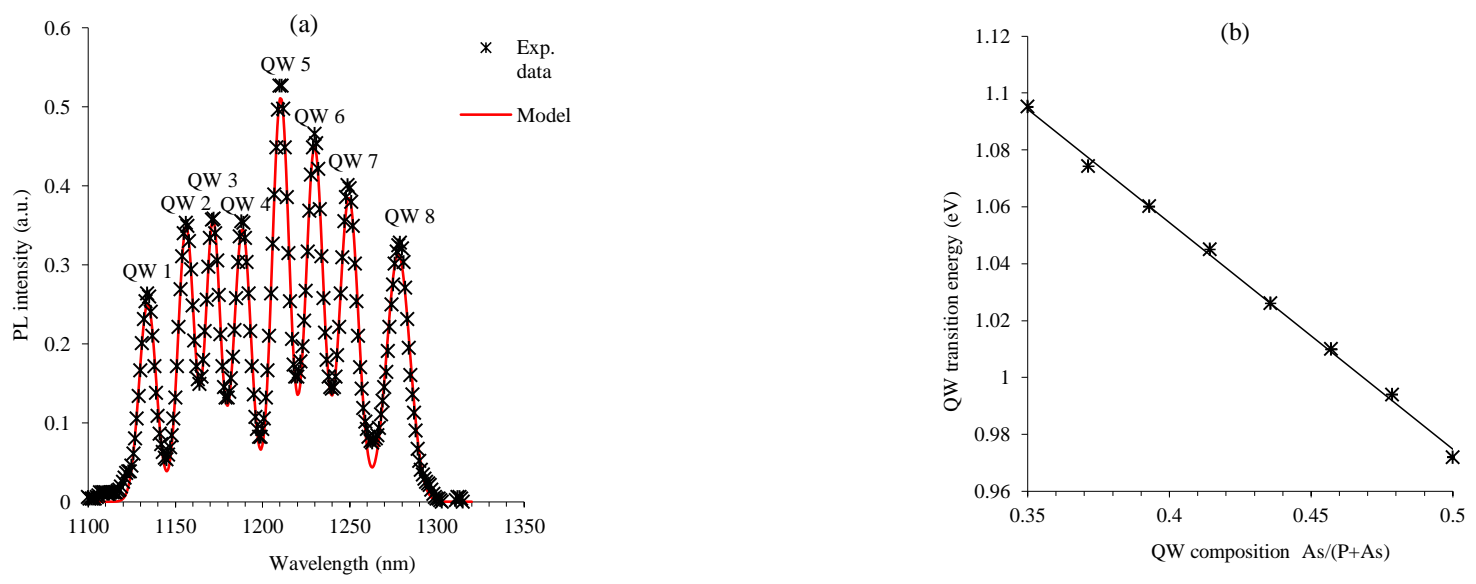

Figure 2. (a) PL spectrum measured at $10 \mathrm{~K}$ with $1064 \mathrm{~nm}$ excitation after epitaxial growth (sample structure shown in fig. 1). Black symbols $(\star)$ : experimental data; full line (red): best-fit with the model described in text. (b) PL line energy versus QW composition. The full line in (b) is a linear best-fit to the PL energies.

procedure was used to extract the spectral parameters from this PL spectrum. This procedure assumes a Gaussian shape for each line, with adjustable central wavelength, full width at half maximum (FWHM) and amplitude for each peak. The calculated PL 
intensity as a function of wavelength $I_{P L}(\lambda)$ is given by :

$$
I_{P L}(\lambda)=\sum_{i=1}^{8} A_{i} \cdot \exp \left(-\frac{\left(\lambda-\lambda_{i}\right)^{2}}{2 \cdot F W H M_{i}^{2}}\right)
$$

where, for each of the eight PL lines, $A_{i}$ denotes the amplitude, $\lambda_{i}$ the central wavelength and $F W H M_{i}$ the full width at half maximum.

The agreement between the experimental data and best-fit procedure is acceptable as shown in figure 2-a (determination coefficient $R^{2}=0.997$ ). The first observation is that a single transition from each QW contributes to each line. This can be explained by the light hole $(\mathrm{LH})$ to heavy hole $(\mathrm{HH})$ energy levels separation in the $\operatorname{InAs}_{x} \mathrm{P}_{1-x}$ QWs induced by compressive stress, which was estimated to be of the order of $0.1 \mathrm{eV}[12,13]$. Only transitions from the electron to the $\mathrm{HH}$ level are observed, the LH level lying too high in energy to significantly contribute to the PL spectrum. The second observation is the sharpness of the PL lines. The FWHM of each line is approximately $4.8 \mathrm{~nm}(4.2$ $\mathrm{meV}$ ) for QW1 - QW7, and $6.7 \mathrm{~nm}(5.1 \mathrm{meV})$ for QW8. The slightly higher FWHM for QW8 may be due to the proximity of the buffer / substrate interface, with some residual impurities. Altogether, the FWHM values are indicative of very high-quality material $[12,13]$. The third observation is the almost linear variation of the QW transition energies versus QW composition (figure 2-b).

The fluctuations of the PL line wavelength across the 2" wafer were less than $1 \mathrm{~nm}$. The short-scale fluctuations (calculated over distances of $30 \mu \mathrm{m}$ ) were smaller than 0.2 $\mathrm{nm}$ for QW1 - QW7, and $0.4 \mathrm{~nm}$ for QW8. These fluctuation values were used to estimate the standard deviation on the QW transitions energies, giving $0.2 \mathrm{meV}$ for QW1 - QW7 and $0.4 \mathrm{meV}$ for QW8. Taking into account these small values, error bars were not displayed on the data of figure 2-b.

Figure 3 shows the evolution of the integrated PL intensities of the QW lines measured on the reference sample, along a $30 \mu \mathrm{m}$ wide line-scan starting at a cleaved edge. This plot will be used as the reference for similar line-scans measured across etched ridges in the following section.

\section{RIE results}

Figure 4 shows a schematic diagram of the excitation laser beam scanned across the etched ridge to produce $\mu \mathrm{PL}$ line-scans. We first observe in figure 5 the superposition of spectra recorded from the reference (un-etched) sample and samples etched by the two different processes. The spectra for the etched samples were taken at the center of $50 \mu \mathrm{m}$ wide stripes, as far as possible from the influence of the etched sidewalls. The PL lines for the etched samples have a higher intensity than for the reference sample. The origin for the higher intensity observed in the un-etched region of the samples was investigated by performing the entire process ( $\mathrm{SiN}_{x}$ mask deposition, exposure to the RIE plasma, mask removal) on samples without stripe definition. The step causing the increase in PL intensity appears to be the $\mathrm{SiN}_{x}$ mask deposition and removal. The $\mathrm{SiN}_{x}$ layer was kept in the HF solution long enough to etch the entire thickness, but we could 


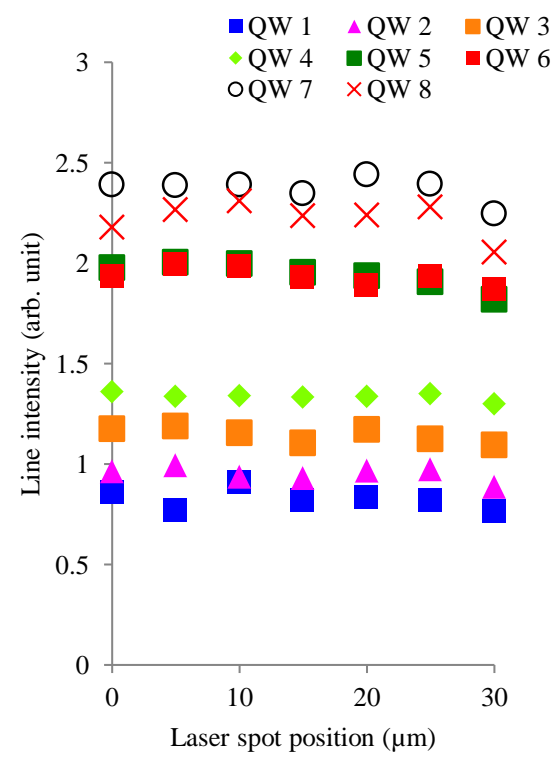

Figure 3. Integrated intensity of the PL lines measured along a $30 \mu \mathrm{m}$ line-scan from the cleaved edge on the reference sample. The cleaved edge is located at the position $0 \mu \mathrm{m}$.

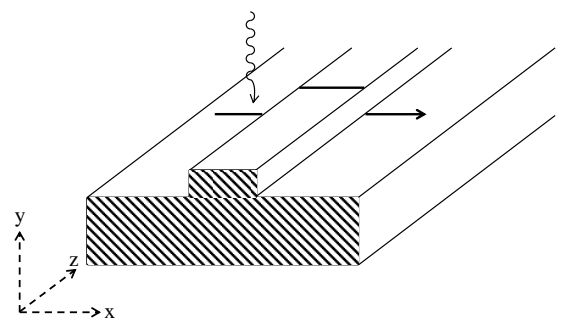

Figure 4. Schematic diagram showing the $\mu \mathrm{PL}$ excitation laser beam scanned across the etched ridge. The dark line with an arrow indicates the line-scan performed along the $x$ direction.

not check for the possible presence of a residual very thin passivation layer (1 or $2 \mathrm{~nm}$ ). We will come back to this in section 5. Small blue shifts of the PL lines also appear for the two etched samples compared to the reference sample, which we will also discuss later.

Next, we discuss the results obtained from $\mu \mathrm{PL}$ line-scans recorded across a $30 \mu \mathrm{m}$ wide stripe etched with the two processes. The integrated PL intensities and spectral positions (peak wavelengths) are shown in figure 6. These data were obtained with the same best-fit procedure as described for the analysis of the spectrum on the reference sample. We checked that the determination coefficient was always higher than 0.95. Integrated intensities for each line were computed using the amplitude and FWHM parameters produced by the best-fit procedure, without any need for baseline subtraction. The integrated intensities (figures 6-a and 6-c) show a gradual increase 


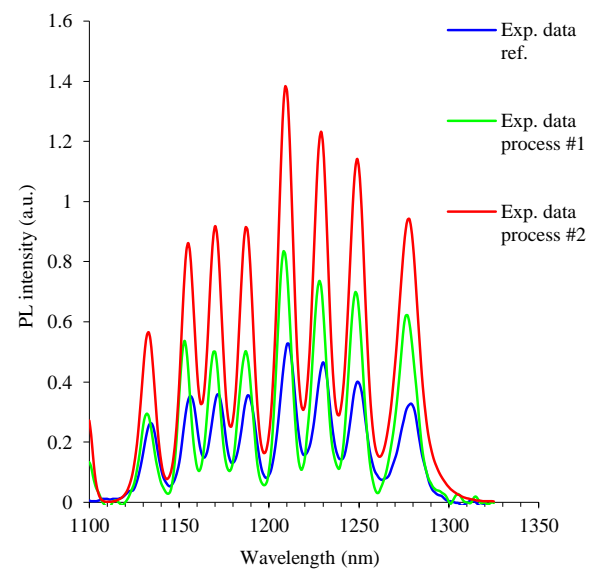

Figure 5. PL spectrum measured at $10 \mathrm{~K}$ with $1064 \mathrm{~nm}$ excitation on the reference sample after epitaxial growth (blue curve), on the sample after etching with process \#1 (green curve) and on the sample after etching with process \#2 (red curve). The spectra for the etched samples were measured at the center of a $50 \mu \mathrm{m}$ wide stripe.

from the edges to the center, with the same trend for all QWs. This intensity increase is not related to the spatial resolution of the $\mu \mathrm{PL}$ setup, which is in the $\mu \mathrm{m}$ range. The peak wavelengths (figures 6-b and 6-d) indicate a detectable blue shift for QW1 and QW2. This blue shift remains constant, within the spectral resolution, throughout the ridge. The blue shifts are illustrated in figure 7 , as a function of the depth location for each QW. Similar trends were observed for $50 \mu \mathrm{m}$ wide ridges.

\section{Discussion}

\subsection{PL lineshapes}

We first note that the evolution of the peak positions during etching of the stripes is a homogeneous effect, in the sense that the spectral peak positions appear nearly constant across the stripes etched with both processes. A trend for a small blue shift is observed for all QWs (figure 7), with a maximum for the QWs closest to the surface. The PL linewidths displayed in figure 8 show a tendency to decrease after the etching processes, again homogeneously (no observation of position-dependent FWHM changes across etched stripes). This FWHM reduction is more pronounced for process \#1, and for QW1 and QW2, located closest to the surface, and also for QW8, located closest to the substrate. RIE in general is expected to increase the PL linewidths [14] through the generation of some disorder at QW interfaces. The small blue shift (with a maximum value for QW1 and QW2), together with the linewidth reduction, may suggest a reduction of the quantum confined Stark effect [15] caused by the residual built-in electric field present in the sample. The residual built-in electric field is the result of the Fermi level pinning at the sample surface after MBE growth. One should note that the residual built-in electric field causing the quantum confined Stark effect 


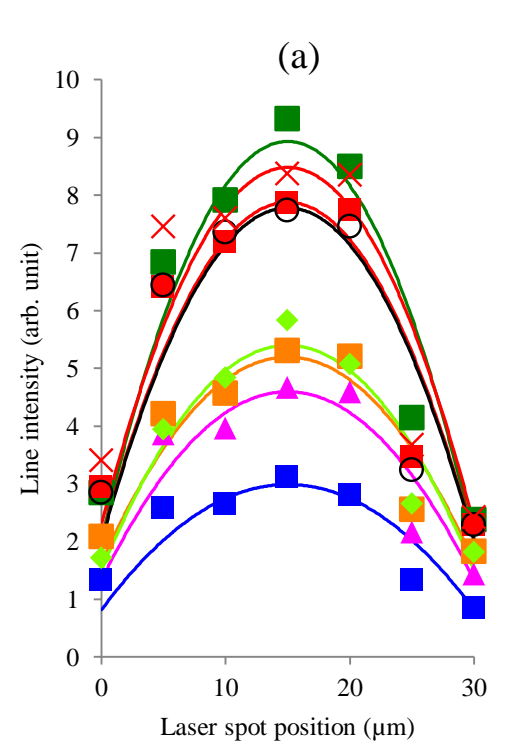

(c)

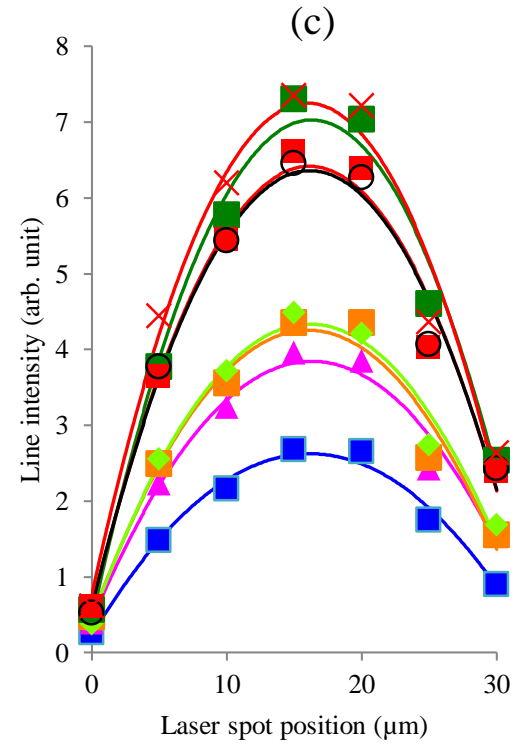

- $\mathrm{QW} 1$

$\triangle \quad$ QW 2

- QW 3

- QW 4

- QW 5

- QW 6

- QW 7

$\times \quad$ QW 8

- Ref. QW 1

Ref. QW 2

- Ref. QW 3

- Ref. QW 4

- Ref. QW 5

- Ref. QW 6

Ref. QW 7

Ref. QW 8

- QW 1

$\triangle \quad$ QW 2

- QW 3

QW 4

- QW 5

- QW 6

- QW 7

$\times \quad$ QW 8

Ref. QW 1

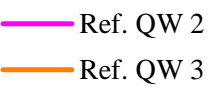

Ref. QW 4

Ref. QW 5

Ref. QW 6

Ref. QW 7

Ref. QW 8 (b)

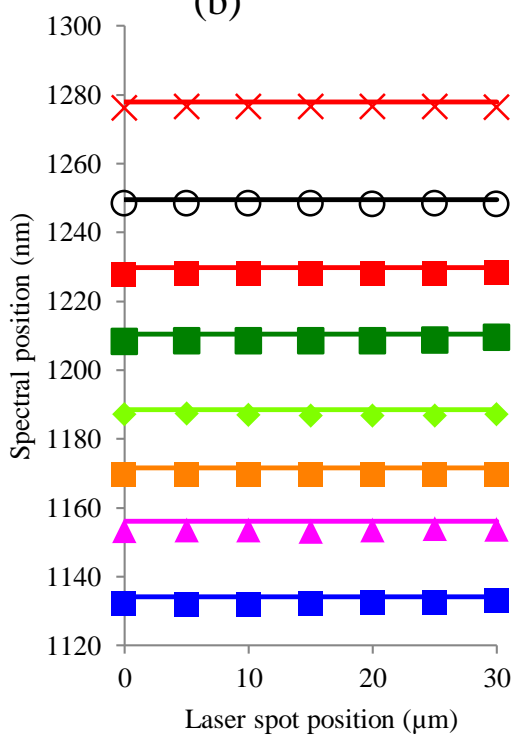

(d)

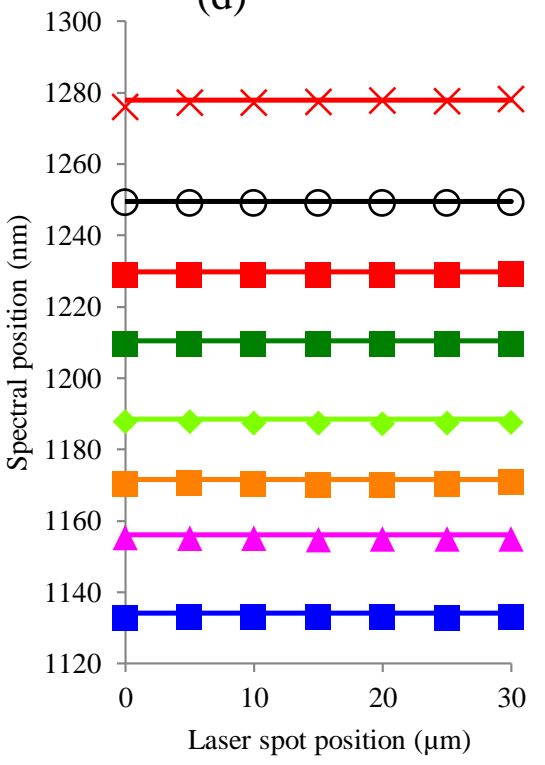

Figure 6. Spectral characteristics of the PL lines measured across a $30 \mu \mathrm{m}$ wide ridge. (a) Process \#1, integrated intensities. (b) Process \#1, spectral positions. (c) Process \#2, integrated intensities. (d) Process \#2, spectral positions. The reference lines in (b) and (d) indicate the spectral position of the corresponding QW measured on the un-etched reference sample (cf. fig. 2). Full lines in (a) and (c) are the best-fits to the experimental line intensity data using the model described in the text. 


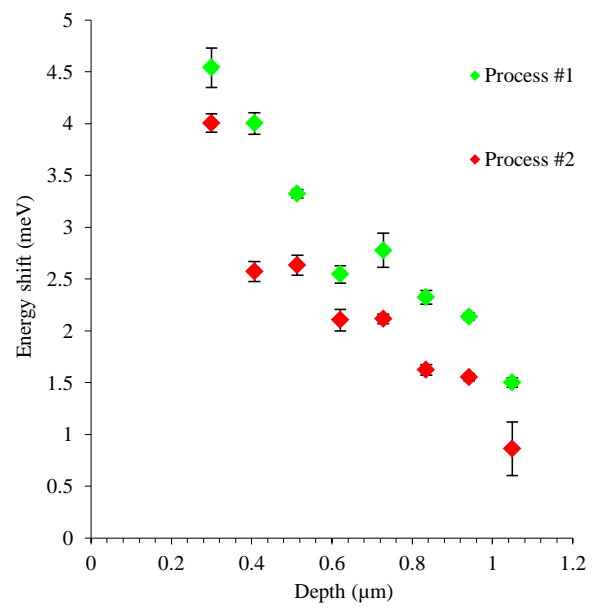

Figure 7. Energy shift of the QW transitions produced by the two etching processes.
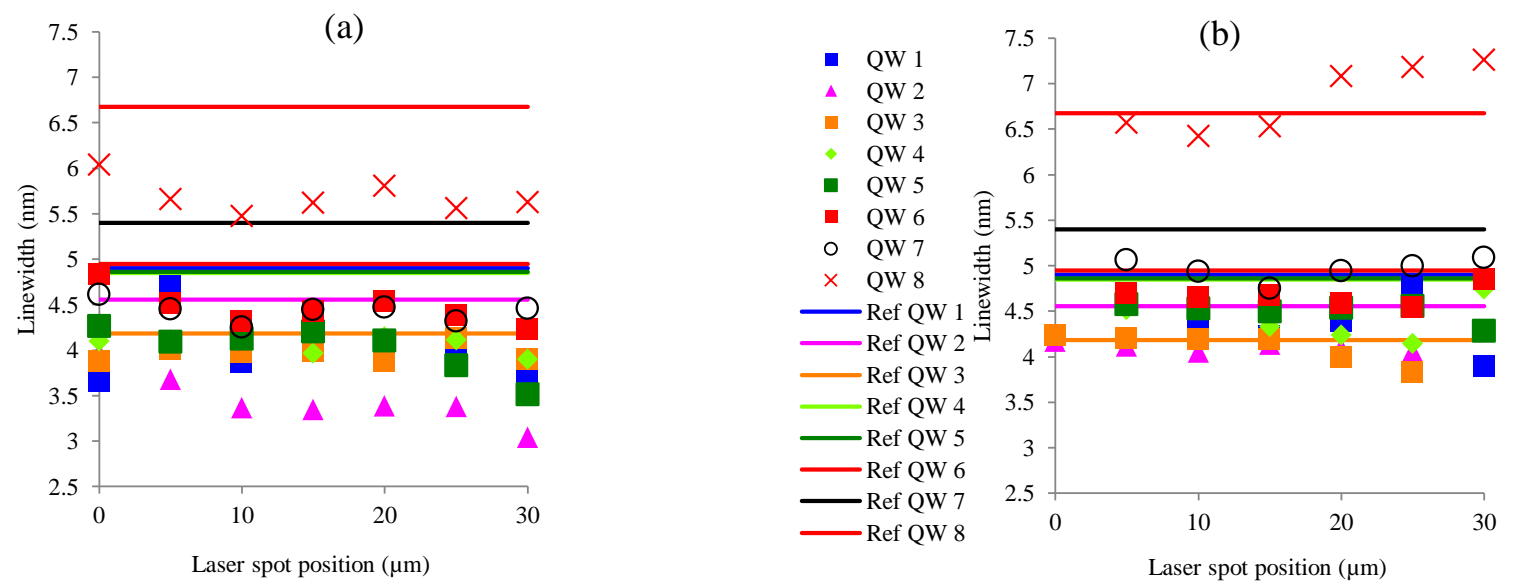

Figure 8. PL linewidths measured across $30 \mu \mathrm{m}$ wide stripes etched with the two etching processes. (a) process \#1; (b) process \#2.

is actually also conditioned by the presence of the photo-generated carriers due to PL excitation and the non-radiative recombination at the surface. Thus we propose that a passivation of the InP surface occurs due to the $\mathrm{SiN}_{x}$ mask deposition and remains even after removal of this mask layer by the HF etching. This surface passivation reduces the SRV, increasing the PL intensities (as illustrated e.g. in the paper by Casey and Buehler [16]), and simultaneously reducing the built-in electric field.

\subsection{PL intensities}

As shown in figures 6 and 9 (for $50 \mu \mathrm{m}$ wide stripes), integrated PL intensities vary strongly across etched stripes. The evolution of the PL intensities can be described by the superposition of a homogeneous change, affecting the overall intensities in the unetched areas, and an inhomogeneous change which results in the intensity distribution 

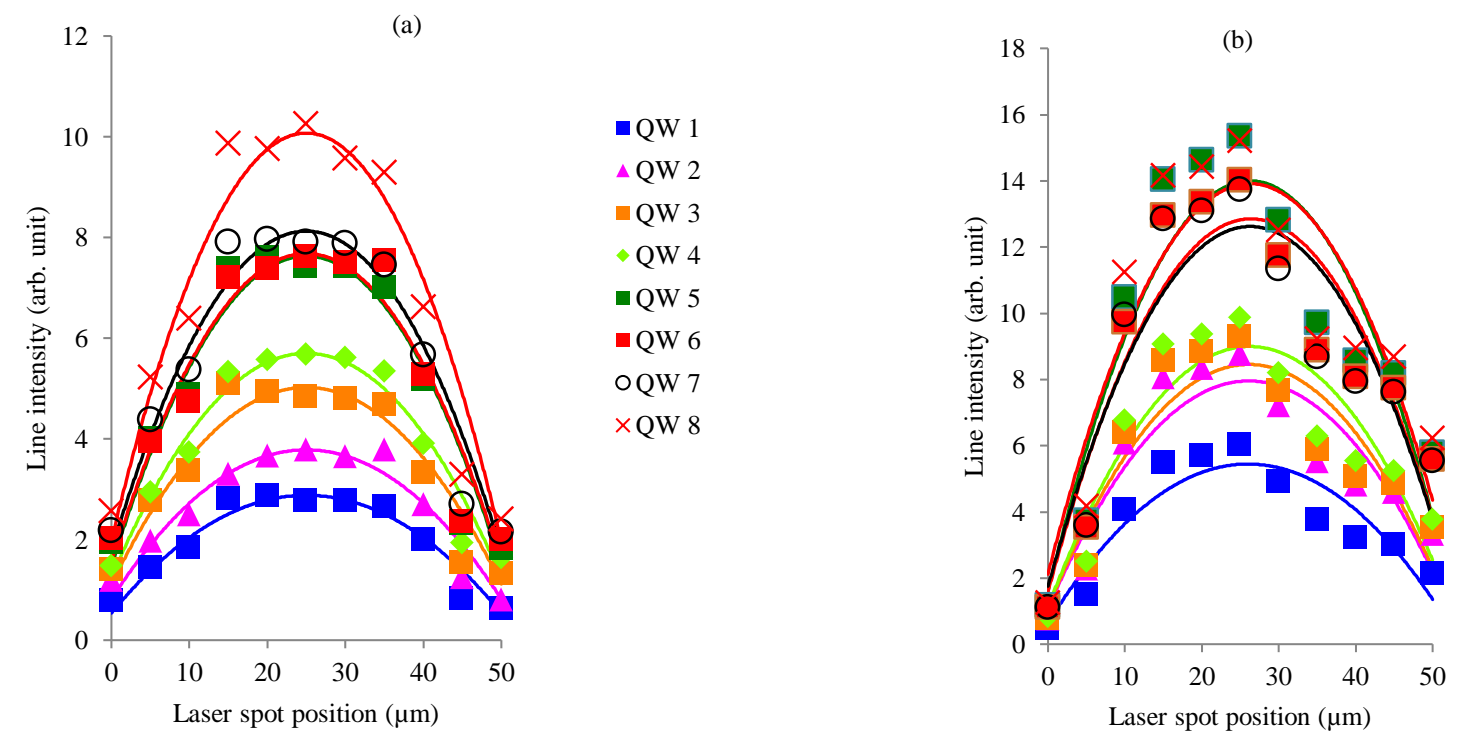

Figure 9. PL integrated line intensities measured across a $50 \mu \mathrm{m}$ wide ridge etched using (a) process \#1, and (b) process \#2. Full lines are the best-fits to the experimental line intensity data using the model described in the text.

Table 1. Maximum of the integrated PL intensity (arb. units) for each QW, for both types of stripes and for both etching processes.

\begin{tabular}{ccccc}
\hline QW & Etching process \#1 & \multicolumn{2}{c}{ Etching process \#2 } \\
\hline & $30 \mu \mathrm{m}$ & $50 \mu \mathrm{m}$ & $30 \mu \mathrm{m}$ & $50 \mu \mathrm{m}$ \\
\hline 1 & 3.1 & 2.9 & 2.7 & 6.0 \\
2 & 4.7 & 3.8 & 4.0 & 8.8 \\
3 & 5.3 & 5.1 & 4.4 & 9.3 \\
4 & 5.8 & 5.7 & 4.5 & 9.9 \\
5 & 9.3 & 7.6 & 7.3 & 15.3 \\
6 & 7.9 & 7.6 & 6.6 & 14.0 \\
7 & 7.7 & 8.0 & 6.5 & 13.7 \\
8 & 8.4 & 10.2 & 7.3 & 15.2 \\
\hline
\end{tabular}

across the stripes. Comparison of figures 6 and 9 with figure 3 (integrated intensity profiles on a line-scan measured from a cleaved edge on the reference sample) illustrates these two different trends. The homogeneous change could be the result of the surface passivation produced by the $\operatorname{SiN}_{x}$ mask deposition and removal process whereas the intensity variation across the stripes (inhomogeneous change) could be the result of the RIE process itself. Table 1 summarizes the maximum of the integrated PL intensities recorded for the different QWs in both types of stripes, and for both etching processes. For process \#1, on average, the maxima of the PL intensities are similar for $50 \mu \mathrm{m}$ 
wide stripes as compared to $30 \mu \mathrm{m}$ wide stripes. For process \#2, the maxima of the PL intensities are approximately twice as high for $50 \mu \mathrm{m}$ wide stripes as compared to 30 $\mu \mathrm{m}$ wide stripes. For the $50 \mu \mathrm{m}$ stripes, PL intensities are higher by $80 \%$ after process \#2 compared to process \#1.

The spatial variation of the PL intensity across the etched stripes could originate from three kinds of mechanisms. \#1: non-radiative recombination at the vertical etched sidewalls (due to defects introduced on these surfaces by the etching process), \#2: non-radiative recombination due to point defects diffusing laterally from the etched walls (these defects would result from interaction with the etching plasma), and \#3: local modification of the electronic structure, for example crystal deformation due to mechanical stress.

A theoretical description of the effect of non-radiative recombination at the limiting surface of a semi-infinite semiconductor has been proposed by Van Roosbroeck [17] and applied experimentally to various cases for determining the surface recombination velocity at various kinds of structures [18]. In this description, the length scale for the spatial variation of the PL line intensities is determined by the diffusion length of photo-generated carriers. Spatially resolved optical measurements, similar to what is described here, have been used to determine minority carrier diffusion length in various III-V semiconductor-based materials and heterostructures [19]. The diffusion lengths obtained are typically in the range of a few microns for the highest quality materials $[19,20]$. In our measurements, the PL line intensities vary over lengths of 15 to $20 \mu \mathrm{m}$ (figures 6 and 9), significantly longer than the diffusion lengths reported; therefore, the non-radiative recombination at the vertical etched sidewalls alone cannot account for the luminescence intensity profiles recorded. Non-radiative recombination at some point defects diffusing laterally from the vertical etched walls, as a result of the interaction of the etched material with the plasma, would also require defect diffusion lengths of at least 15 to $20 \mu \mathrm{m}$ to explain our observations, which is not realistic either. Therefore, we suggest that the reduction of the PL intensities is caused predominantly by a local modification of the electronic band structure within the QWs, resulting from a crystal deformation due to mechanical stress introduced by the etching process. The occurrence of mechanical stress during RIE of stripes in GaAs and InP has been demonstrated recently by Landesman et al [5]. The origin of this mechanical stress could be related to the ion bombardment taking place during exposure to the etching plasma. As previously described in [5], the resulting crystal deformation consists mainly of a hydrostatic volume compression taking place at the vertical etched sidewalls and extending several microns inside the stripes. Such volume compression should change the band gap, which was actually observed by both $\mu \mathrm{PL}$ and spatially resolved cathodoluminescence. The fact that the PL peak positions do not significantly vary near the edges of the stripes in the present study indicates that this local crystal deformation close to the etched sidewalls cannot be dominant. However, another crystal deformation contribution must be taken into account: due to the creation of free surfaces at the vertical etched sidewalls, the initial biaxial compressive stress within the $\operatorname{InAs}_{x} \mathrm{P}_{1-x}$ QWs has to relax partially. This 
kind of relaxation has been investigated in detail both experimentally and theoretically $[21,22]$. In our sample, it should consist mainly of a uniaxial tensile deformation perpendicular to the stripe length, and parallel to the wafer surface ( $x$ direction in figure 4). This kind of relaxation can extend over distances up to several $\mu \mathrm{m}$ away from the stripe edge.

The resulting local electronic structure within the QWs, in particular the location of the different hole sub-bands and sub-band mixing, should affect both PL intensities and peak positions. A quantitative computation requires solving the local electronic band structure for the QWs taking into account a coupling with the elastic crystal deformation describing the relaxation phenomenon as well as the effect of RIE. Surface non-radiative recombination should also be included. This is outside the scope of this paper and will be considered in the future. A simple approach is presented below.

To emphasize the variations of PL intensities in the etched stripes we have performed a best-fit procedure to the experimental data. As an analytical model for the dependence of the PL intensity versus the distance to the edges, we have tried on the first hand a formula with exponential variation (such as would result from non-radiative recombination at the etched sidewalls $[18,17])$ and on the second hand a formula with erf $c$-type variation (which would quantify the impurity concentration variation near the etched sidewalls as a solution to the first Fick's law [23]). These are two extreme physically possible assumptions for the observed variations of the PL intensities. However, both models yield acceptable agreement with the experimental data. Our choice here is to illustrate the best-fit procedure using the first choice, based on the formula:

$$
I_{P L}(x)=A[1-B \exp (-\alpha \cdot x)-B \exp (-\alpha(W-x))]
$$

where $I_{P L}(x)$ is the PL intensity measured at location $x$ within the stripe. $A$ and $B$ are constants: $A$ holds for the PL intensity in an area infinitely far from the etched sidewalls, and $A \cdot(1-B)$ is the PL intensity which would be measured exactly at the etched sidewalls (in other words, $B$ is the fraction of the intensity reduction caused by the sidewalls). $W$ is the stripe width. $\alpha$ is a coefficient accounting for the intensity variation from both stripe edges. The best-fit curves are shown in figure 6 (a) and (c) for $30 \mu \mathrm{m}$ wide stripes and in figure 9 (a) and (b) for $50 \mu \mathrm{m}$ wide stripes. As mentioned earlier the fact the PL line intensities vary over lengths of 15 to $20 \mu \mathrm{m}$ away from the etched sidewalls tells us that the coefficient $\alpha$ cannot be related to non-radiative surface recombination as would suggest the analytical model we used for the best-fit procedure. The observations on the PL line intensities demonstrate a strong spatial modulation driven by the different effects suggested. Comparison of the evolution of the PL line intensities across 30 and $50 \mu \mathrm{m}$ wide stripes, etched with the two different processes, indicates a significant difference between the two etching processes. From the potential mechanisms contributing to PL intensity variations, biaxial stress relaxation associated with the formation of the sidewall free surfaces should be similar for both etching processes. However, one has to consider the stress generation by the etching 
process itself, as well as the non-radiative recombination at the sidewalls, and these two factors are certainly very different for the two etching processes. Solving this issue requires the complete numerical quantitative approach which will be addressed in a separate article.

\section{Conclusion}

The results described illustrate the potential of low temperature $\mu \mathrm{PL}$ measurements for a careful assessment of microstructures based on QW designs. The details of our study reveal the complex physics resulting from the etching processes. Thanks to the high quality of the QW samples, the PL lines for each QW could be measured individually with high accuracy and line-scans were performed across ridge stripes etched with different RIE processes. Two trends of changes of the integrated PL intensities were observed: one homogeneous and one inhomogeneous. For the homogeneous change we proposed a mechanism of surface passivation by the $\mathrm{SiN}_{x}$ mask, which persists after the removal of this mask. This surface passivation mechanism may be due to a remaining very thin layer $(1-2 \mathrm{~nm})$ after removal of the $\mathrm{SiN}_{x}$ mask, or to the effect of the $\mathrm{HF}$ etching ( $\mathrm{H}$ passivation ?). For the inhomogeneous change, we proposed three origins: non-radiative recombination (mainly at the vertical etched sidewalls), compressive volume change near the etched sidewalls generated by ion bombardment from the plasma, and uniaxial relaxation of the built-in compressive stress in the asgrown QWs. A significant difference in the behavior for PL line intensities was observed between the two studied etching processes. Whereas for the $30 \mu \mathrm{m}$ wide stripes, the PL intensities are similar for both etching processes, for the $50 \mu \mathrm{m}$ wide stripes these intensities are approximately $80 \%$ higher for process \#2 as compared to process \#1. A detailed quantitative analysis is required to explain this difference. Uniaxial relaxation of the built-in compressive stress in the QWs should be identical in magnitude for both etching processes. The other two mechanisms (compressive volume change near the etched sidewalls, non-radiative recombination at the sidewalls) may lead to strong differences between the two etching processes. At this stage, we suggest that the different magnitude of these mechanisms explains the difference observed on the PL intensities. The temperature involved during the etching process (higher for process \#2 than for process \#1), or the amount of hydrogen present (higher for process \#1) might be some of the origins. Future work will perform quantitative simulations of the PL line intensities, using a coupled model taking into account local electronic band structure within the QWs, the associated optical transitions, and the effect of elastic strain relaxation. The electronic band structure calculation will be performed using either a full ab-initio approach or a more simple $k \cdot p$ hamiltonian description.

The spectral shifts of the PL lines display a homogeneous behavior. These line shifts appear constant with a high accuracy for each QW across the etched stripes, suggesting that the mechanisms responsible for PL intensity variations do not produce any spectral shift, or that some compensation takes place leading to overall negligible peak shift. 


\section{Acknowledgments}

JPL and CL acknowledge support from the NanoRennes platform, affiliated to RENATECH+, the French national facilities network for micro-nanotechnology. RRL and NIG acknowledge support from the Natural Sciences and Engineering Research Council of Canada (NSERC), Grant RGPIN-2018-04015 and RGPAS-2018-522624. JJ was funded by Junta de Castilla y León (Project No. VA283P18) and MINECO (Project No. RTI2018-101020-B-I00).

\section{References}

[1] Mohseni P K, Rodrigues A D, Galzerani J C, Pusep Y A and LaPierre R R 2009 J. Appl. Phys. 106124306

[2] Zhang Y, Xu J, Xiang Q, Li H, Pan Q and Xu P 2009 J. Phys. Chem. C 113 3430-3435

[3] Wegscheider W, Schedelbeck G, Abstreiter G, Rother M and Bichler M 1997 Phys. Rev. Lett. 79 $1917-1920$

[4] Kunert B, Guo W, Mols Y, Tian B, Wang Z, Shi Y, Van Thourhout D, Pantouvaki M, Van Campenhout J, Langer R and Barla K 2016 Appl. Phys. Lett. 109091101

[5] Landesman J P, Fouchier M, Pargon E, Gérard S, Rochat N, Levallois C, Mokhtari M, PagnodRossiaux P, Laruelle F, Petit-Etienne C, Bettiati M, Jiménez J and Cassidy D T $2020 \mathrm{~J}$. Appl. Phys. 128225705

[6] Vigneron P, Joint F, Isac N, Colombelli R and Herth E 2018 Microelectron. Eng. 202 42-50

[7] Booker K, Mayon Y O, Jones C, Stocks M and Blakers A 2020 J. Vac. Sci. Technol. B 38012206

[8] Renaud V, Petit-Etienne C, Barnes J P, Bisserier J, Joubert O and Pargon E 2019 J. Appl. Phys. 126243301

[9] de Lafontaine M, Pargon E, Petit-Etienne C, Gay G, Jaouad A, Gour M J, Volatier M, Fafard S, Aimez V and Darnon M 2019 Sol. Energy Mater. Sol. Cells 195 49-54

[10] Pargon E, Gay G, Petit-Etienne C, Brihoum M, Bizouerne M, Burtin P and Bamola S 2016 Plasma etching processes for the integration of InP based compounds on $200 \mathrm{~mm} \mathrm{Si}$ wafer for photonic applications SPIE 9782, Advanced Etch Technology for Nanopatterning V (San Jose, CA, United States) p $97820 \mathrm{C}$

[11] Haapamaki C M 2012 Growth of InAs/InP Nanowires by Molecular Beam Epitaxy Ph.D. thesis McMaster University Hamilton, ON Canada p. 20-26

[12] Monier C, Vilela M F, Serdiukova I and Freundlich A 1998 J. Cryst. Growth 188 332-337

[13] Hou H Q and Tu C W 1994 J. Appl. Phys. 75 4673-4679

[14] Djie H S, Arokiaraj J, Mei T, Tang X H, Ang L K and Leong D 2003 J. Vac. Sci. Technol. B 21 L1

[15] Vina L, Mendez E E, Wang W I, Chang L L and Esaki L 1987 J. Phys. C: Solid State Phys. 20 $2803-2815$

[16] Casey H C and Buehler E 1977 Appl. Phys. Lett. 30 247-249

[17] Van Roosbroeck W 1955 Journal of Applied Physics 26 380-391

[18] Mendis B G and Bowen L 2011 J. Phys. Conf. Ser. 326012017

[19] Luber D R, Bradley F M, Haegel N M, Talmadge M C, Coleman M P and Boone T D 2006 Appl. Phys. Lett. 88163509

[20] Fiore A, Rossetti M, Alloing B, Paranthoen C, Chen J X, Geelhaar L and Riechert H 2004 Phys. Rev. B $\mathbf{7 0} 205311$

[21] Jain S C, Willander M and Maes H 1996 Semicond. Sci. Technol. 11 641-671

[22] Fierling G, Buchheit M, Letartre X, Gendry M, Viktorovitch P, Sidoroff F and Lainé J P 1997 Appl. Phys. Lett. 71 1516-1518

[23] Willoughby F W 1978 Rep. Prog. Phys. 41 1965-1705 